$63^{\text {ème }}$ Congrès de la SFCO, 02010 (2015)

DOI:10.1051/sfco/20156302010

(C) Owned by the authors, published by EDP Sciences, 2015

\title{
COMMUNICATION
}

\section{Corps adipeux de la bouche en chirurgie orale : intérêts et perspectives}

\author{
Carles S, Labadie M-P, Lhomme A, Courtois B \\ Département de Chirurgie Orale, CHU de Toulouse
}

\section{Introduction}

Depuis sa première utilisation (Egyedi 1977) le Corps Adipeux de la Bouche (CAB) représente un élément anatomique d'intérêt clinique en chirurgie orale. Ses caractéristiques anatomo-physiologiques ont permis d'élargir ses indications à mesure des avancées chirurgicales, notamment en chirurgie préimplantaire. Ce travail présente une actualisation des données de la littérature sur l'intérêt du CAB en chirurgie orale et identifie des perspectives cliniques à approfondir.

Matériel et méthode

Nous avons réalisé une revue de la littérature sur Pubmed en s'appuyant sur la méthodologie des revues systématiques, en utilisant le MeSH avec l'équation : ("Buccal Fat Pad”' AND “Oral surgery” [Mesh] OR “Maxillofacial surgery” [Mesh] OR “Oral disease” [Mesh]).

Les critères de sélection des articles étaient: langue anglaise ou française, publication entre 1980 et 2014 et résumé disponible sur Pubmed.

Résultats

38 articles ont été retenus sur 98 sélectionnés.

Un premier résultat émerge concernant les indications «traditionnelles » que représentent : la fermeture de communication bucosinusienne (CBS) et les reconstructions de perte de substance postchirurgicale. Les 19 études retenues montrent un taux de succès > $95 \%$ (utilisation du CAB en lambeau pédiculé, observation d'une épithélialisation complète en 4 à 6 semaines). La chirurgie préimplantaire constitue le second domaine d'application du CAB. 5 études mettent en évidence l'intérêt de ses propriétés de cicatrisation lors de sinus lift (fermeture latérale du site ou réparation perforation de membrane) ainsi que lors de greffe d'apposition. 1 étude souligne les perspectives d'utilisation du CAB dans ce cadre, en lambeau libre avec 19 succès sur 20 cas de couverture de greffe en apposition. Le dernier domaine d'intérêt représente l'utilisation du CAB comme source de cellules souches ostéogéniques, potentiellement utiles dans le domaine des greffes osseuses. 4 études in vitro isolent la Fraction Stromale Vasculaire (SVF) du tissus graisseux du CAB et mettent en évidence une augmentation des paramètres indirects de différentiation ostéogénique (Phosphatase Alcaline, Collagène, Calcification de la matrice extracellulaire) lors de sa culture en milieu ostéo-inducteur. L'étude in vivo montre une formation d'os ectopique à 8 semaines d'une implantation sous-cutané chez la souris de cellules de la SVF humaine associées à une protéine recombinante rh-BMP2.

This is an Open Access article distributed under the terms of the Creative Commons Attribution License 4.0, which permits unrestricted use, distribution, and reproduction in any medium, provided the original work is properly cited. 


\section{Conclusion}

Ce travail met en évidence des résultats prometteurs concernant l'utilisation du CAB en chirurgie orale notamment dans le domaine de la chirurgie préimplantaire. Le recours au CAB comme technique de couverture des greffes osseuses doit être approfondie et le développement de l'exploitation des cellules souches de ce tissu graisseux particulier doit être envisagé dans un rapport de réciprocité entre recherche fondamentale et pratique clinique.

Nom et adresse du conférencier

\section{Sébastien CARLES}

UFR Odontologie Toulouse (France)

sebastien.carles31@gmail.com 